\title{
Co/Ni Element Ratio in the Galactic Cosmic Rays between 0.8 and $4.3 \mathrm{GeV} / \mathrm{nucleon}$
}

\author{
S.H. Sposato ${ }^{1}$, L.M. Barbier ${ }^{2}$, W.R. Binns ${ }^{1}$, E.R. Christian ${ }^{2}$, J.R. Cummings ${ }^{1}$, \\ G.A. deNolfo ${ }^{3}$, P.L. Hink ${ }^{1}$, M.H. Israel ${ }^{1}$, R.A. Mewaldt ${ }^{3}$, J.W. Mitchell ${ }^{2}$, \\ S.M. Schindler ${ }^{3}$, R.E. Streitmatter ${ }^{2}$, C.J. Waddington ${ }^{4}$ \\ 'Department of Physics \& McDonnell Center for the Space Sciences, Washington University, St. Louis, MO 63130 \\ ${ }^{2}$ Laboratory for High Energy Astrophysics, Goddard Space Flight Center, Greenbelt, MD 20771 \\ ${ }^{3}$ Space Radiation Laboratory, California Institute of Technology, Pasadena, CA 91125 \\ ${ }^{4}$ School of Physics and Astronomy, University of Minnesota, Minneapolis, MN 55455
}

\begin{abstract}
In a one-day balloon flight of the Trans-Iron Galactic Element Recorder (TIGER) in 1997, the instrument achieved excellent charge resolution for elements near the Fe peak, permitting a new measurement of the element ratio $\mathrm{Co} / \mathrm{Ni}$. The best fit to the data, extrapolated to the top of the atmosphere, gives an upper limit for this ratio of $0.093 \pm$ 0.037 over the energy interval 0.8 to $4.3 \mathrm{GeV} /$ nucleon; because a Co peak is not seen in the data, this result is given as an upper limit. Comparing this upper limit with calculations by Webber \& Gupta [14] suggests that at the source of these cosmic rays a substantial amount of the electron-capture isotope ${ }^{59} \mathrm{Ni}$ survived. This conclusion is in conflict with the clear evidence from ACE/CRIS below $0.5 \mathrm{GeV} /$ nucleon that there is negligible ${ }^{59} \mathrm{Ni}$ surviving at the source. Possible explanations for this apparent discrepancy are discussed.
\end{abstract}

\section{INTRODUCTION}

The Trans-Iron Galactic Element Recorder (TIGER) is a balloon-borne cosmic-ray experiment that utilizes plastic scintillation counters, plastic and aerogel Cherenkov counters, and scintillating optical fiber hodoscopes. With this combination of detectors we have achieved charge resolution of 0.23 charge units $(\mathrm{cu})$ for elements near the Fe peak for energies < $4.3 \mathrm{GeV} /$ nucleon. This resolution has enabled a new measure of the $\mathrm{Co} / \mathrm{Ni}$ abundance ratio using data from the one-day balloon flight from Fort Sumner, NM in September 1997.

The Co/ $\mathrm{Ni}$ measurement is interesting because it can put limits on the time between the nucleosynthesis and acceleration of cosmic rays [8]. ACE/CRIS has measured isotopes of $\mathrm{Co}$ and $\mathrm{Ni}$ in the energy interval $150 \mathrm{MeV} /$ nucleon - $500 \mathrm{MeV} /$ nucleon. That measurement found that ${ }^{59} \mathrm{Ni}$, which decays only by electron capture (half-life $76,000 \mathrm{yr}$ ), has all decayed to ${ }^{59} \mathrm{Co}$, implying that acceleration occurred more than $10^{5}$ years after nucleosynthesis [15]. At higher energies isotope data are not available, but elemental abundances can put constraints on the decay of ${ }^{59} \mathrm{Ni}$. Engelmann et al. [2] measured a $\mathrm{Co} / \mathrm{Ni}$ ratio of $\approx 0.12$ near $1 \mathrm{GeV} /$ nucleon. Previous balloon measurements of this ratio, extrapolated to the top of the atmosphere, have been $0.17 \pm 0.05[1]$ and $0.31 \pm 0.15[3]$.

\section{THE TIGER INSTRUMENT}

The TIGER instrument contains three scintillation counters with wave-length-shifter-bar readout and two Cherenkov detectors: an acrylic radiator $(\mathrm{n}=1.5)$ in a light box and an aerogel radiator $(n=1.04)$ in a light box. (See Figure 1.) There are two scintillators on the top of the stack and one at the bottom. The scintillation counters and the Cherenkov detectors provide charge and velocity measurements of the cosmic rays, provided that pathlength through the instrument can be determined. 
The trajectory of each cosmic ray through the instrument is measured to within a few millimeters using a Coarse Hodoscope and a coded Fine Hodoscope. Each consists of two planes of $1.5 \mathrm{~mm} \mathrm{x}$ $1.5 \mathrm{~mm}$ square scintillating optical fibers, one at the top of the stack and one at the bottom. Each plane has two layers of fibers, one each in the $\mathrm{X}$ and $\mathrm{Y}$ directions. The Coarse Hodoscope determines position to within $8 \mathrm{~cm}$, and the Fine Hodoscope determines position to within $6 \mathrm{~mm}[5,9]$.

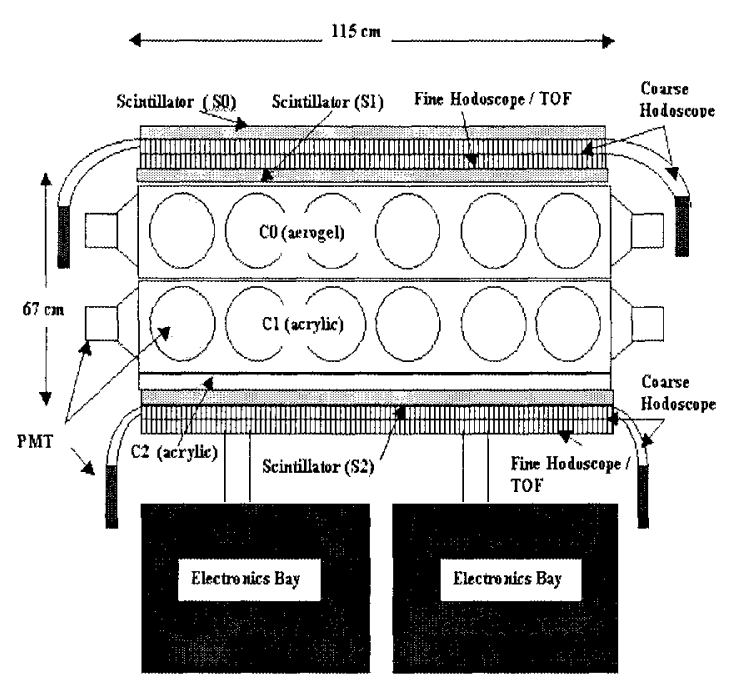

FIGURE 1. Cross-section of the TIGER instrument

\section{DATA ANALYSIS}

There are several steps in the data analysis. The first is a $\sec (\theta)$ correction for variations in pathlength made using the hodoscopes. Mapping corrections are made to the data from each detector to remove areal response variations. Corrections are also made to remove a residual $\theta$ dependence in the data. This dependence is probably due to the change in energy deposition of knock-on electrons with depth in the detector stack. The Z-dependence of the signals from the scintillators and Cherenkov detectors is also determined empirically. Interaction cuts are made to remove particles that changed charge in the detector stack. The difference in charge measured in the top two scintillators is required to be $<0.6 \mathrm{cu}$. Also the difference in charge between the average of the top two scintillators and the bottom scintillator is required to be $<0.6 \mathrm{cu}$.

After these corrections are made, the data are divided into energy intervals. Interval 1 contains $\mathrm{E}<$
$2.7 \mathrm{GeV} /$ nucleon. Interval 2 contains $2.7<\mathrm{E}<4.3$ $\mathrm{GeV} /$ nucleon. The data have an inherent energy dependence in these regions. Empirical fits are made to the energy variations in each region.

A histogram of the data from TIGER in Energy Intervals 1 and $2(\mathrm{E}<4.3 \mathrm{GeV} / \mathrm{n})$ is shown in Figure 2. The charge is determined using the sum of the signals from the three scintillators $\left(Z_{\left(\mathrm{SO}_{\mathrm{S} 1}+\mathrm{S}_{2}\right)}\right)$ and $\mathrm{C} 1$ for the energy fits in Interval 1 , and the sum of the signals from the scintillators and $\mathrm{Cl}\left(\mathrm{Z}_{(\mathrm{SO}+\mathrm{S} 1+\mathrm{S} 2)}+\mathrm{Z}_{\mathrm{Cl}}\right)$ and $\mathrm{C} 0$ for the energy fits in Interval 2.

To calculate the abundance of each element, the histogram is fit with multiple Gaussians, one for each elemental peak. The results from the Gaussian fit are shown superimposed over the scintillator histogram in Figure 2 and Figure 3.

Inefficiencies in the hodoscopes presented the only problem with TIGER during the 1997 Balloon Flight. Multiple hits in the Course Hodoscope produced by knock-on electrons reduced the instrument's ability to detect high-Z, high-energy cosmic rays. The hodoscope bias introduced a charge dependent bias in the TIGER data. To determine this bias, the TIGER data are compared to HEAO-3-C2 data [2] and corrected empirically [10].

The Co/Ni ratio grows with depth in the atmosphere as the $\mathrm{Ni}$ and $\mathrm{Co}$ interact and break up into lighter nuclei. We extrapolated the observed $\mathrm{Co} / \mathrm{Ni}$ to the top of the atmosphere using growth curves for $\mathrm{Mn} / \mathrm{Fe}$ [4]. This method is valid because the values of $\mathrm{Mn} / \mathrm{Fe}$ and $\mathrm{Co} / \mathrm{Ni}$ are both on the order of 0.11 , assuming that the partial cross-section of $\mathrm{Ni}$ to $\mathrm{Co}$ is approximately the same as that of Fe to $\mathrm{Mn}$.

The $\mathrm{Co} / \mathrm{Ni}$ ratio measured by TIGER corrected to the top of the atmosphere is $0.093 \pm 0.037$ (Table 1). In the calculation of this number it is assumed that the elemental abundance peaks are Gaussian. However, as seen in Figure 3 there is no resolved Co peak, and the $\mathrm{Fe}$ peak appears to have a slight non-Gaussian tail, which could account for the amount of Co inferred from the Gaussian fits. Therefore, the above value of the $\mathrm{Co} / \mathrm{Ni}$ ratio should be taken as an upper limit.

\section{Comparison of $\mathrm{Co} / \mathrm{Ni}$ Measurements}

Figure 4 shows the TIGER Co/Ni ratio compared with other measurements. The lines in Figure 4 are calculations [14] that assumed either that ${ }^{59} \mathrm{Ni}$ had not decayed (solid line) or that it had fully decayed 


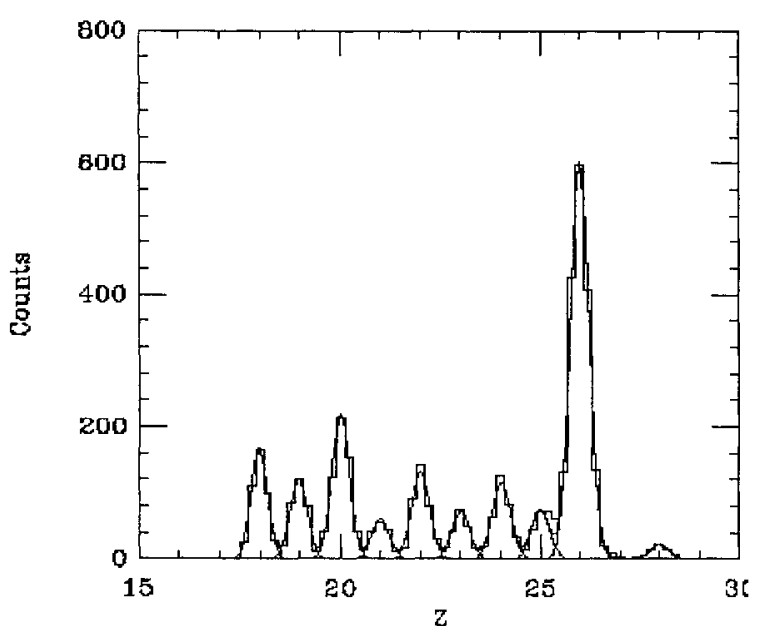

FIGURE 2. Charge histogram for $E<4.3 \mathrm{GeV} / \mathrm{n}$

(dotted line). The TIGER Co/Ni ratio (upper limit) of $0.093 \pm 0.037$, which is lower than previous balloon measurements, is consistent with the HEAO-3-C2 $\mathrm{Co} / \mathrm{Ni}$ ratio for energies between 0.8 and 4.3 $\mathrm{GeV} /$ nucleon. This value is also consistent with the solid Webber \& Gupta [14] line suggesting that the ${ }^{59} \mathrm{Ni}$ at the source has not had time to decay to ${ }^{59} \mathrm{Co}$. At face value, the TIGER measurement and the general trend of the HEAO data suggest that a substantial amount of ${ }^{59} \mathrm{Ni}$ has not decayed, implying a short time delay of $<7.6 \times 10^{4}$ years between nucleosynthesis and acceleration, although the "complete-decay" line is only $2 \sigma$ above the TIGER measurement.

On the other hand, the ACE/CRIS Co/Ni elemental measurement of $0.137 \pm 0.13$ [7] at energies between $150-500 \mathrm{MeV} /$ nucleon is $2 \sigma$ away from the "nodecay" line and $2 \sigma$ away from the "complete-decay" line. We note this interpretation of the elemental $\mathrm{Co} / \mathrm{Ni}$ ratios is not consistent with the isotopic measurement made by ACE/CRIS at $<500$ $\mathrm{MeV} /$ nucleon. The ACE-CRIS mass histograms of $\mathrm{Ni}$ and Co show that at earth there is a substantial amount of ${ }^{59} \mathrm{Co}$ in the GCRs and almost no ${ }^{59} \mathrm{Ni}$. That result indicates that decay of ${ }^{59} \mathrm{Ni}$ to ${ }^{59} \mathrm{Co}$ has occurred at the source, and a time delay of longer than $7.6 \times 10^{4}$ years has elapsed before the particles were accelerated.

\section{Discussion}

Several possible explanations may be suggested for this discrepancy between the indication from our element ratio of $\mathrm{Co} / \mathrm{Ni}$ that ${ }^{59} \mathrm{Ni}$ has not decayed, and the clear ACE/CRIS evidence that it has decayed. The problem most likely lies with the interpretation of the elemental measurements rather than the highly

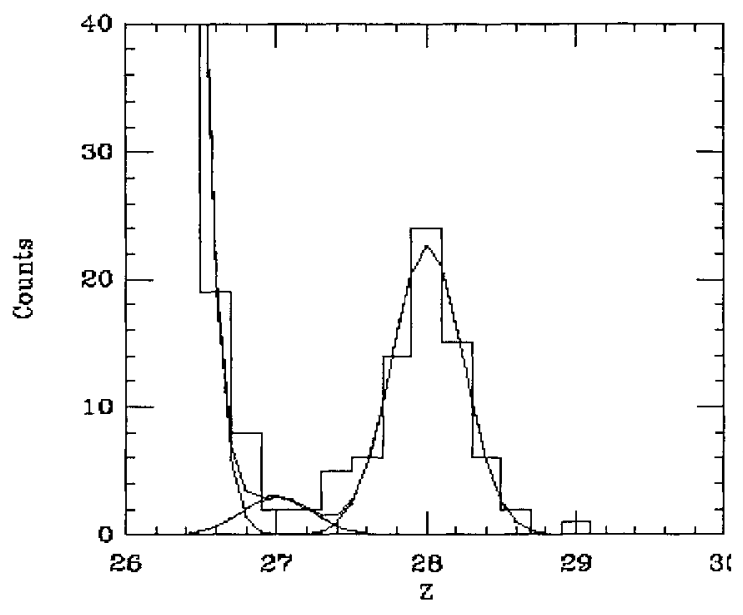

FIGURE 3. Charge histogram for $\mathrm{E}<4.3 \mathrm{GeV} / \mathrm{n}$ in Co-Ni region.

accurate ACE/CRIS isotopic measurement.

Interpretation of the elemental abundance of $\mathrm{Co} / \mathrm{Ni}$ is very model dependent. Different models concerning the amount of Ni produced in SN nucleosynthesis and the interstellar propagation of $\mathrm{Ni}$ and Co would change the interpretation of the $\mathrm{Co} / \mathrm{Ni}$ ratio. For example, a shorter propagation grammage would mean less $\mathrm{Ni}$ could interact in the ISM, producing less $\mathrm{Co}$, and thereby yielding a lower predicted $\mathrm{Co} / \mathrm{Ni}$ ratio. Therefore, a low $\mathrm{Co} / \mathrm{Ni}$ ratio at earth might not mean that there is much Ni back at the source. Because of this, one possible explanation is that the models used in Webber \& Gupta [14] are flawed, using source abundances of $\mathrm{Co}$ and $\mathrm{Ni}$ different from those of the Solar System or incorrect propagation grammages through the ISM.

Another possible explanation is that the total crosssections of $\mathrm{Ni}$ and $\mathrm{Co}$ and the partial cross-section of $\mathrm{Ni}$ to $\mathrm{Co}$ used to produce the curves in Figure 3 are incorrect. The cross-sections used in Webber \& Gupta [14] were measured at $600 \mathrm{MeV} /$ nucleon using a $\mathrm{CH}_{2}$ $\mathrm{C}$ target subtraction technique to determine the crosssection in Hydrogen. More recently, Webber et al. [13] have made a measure of the cross-section of ${ }^{58} \mathrm{Ni}$ to $\mathrm{Co}$ using a $\mathrm{H}$ target with thickness approximating the amount of $\mathrm{H}$ traversed by GCR in the ISM. The new ${ }^{58} \mathrm{Ni}$ to Co partial cross-section of $116.8 \pm 5.94$ mbarn is consistent with the 1990 cross-section of $119.1 \pm 7.5$ mbarn. This new measure of the partial cross-section was again made at energies between 400 and 650 $\mathrm{MeV} / \mathrm{n}$. It is possible that the cross-sections of $\mathrm{Ni}$ and Co need to be measured in the $\mathrm{GeV} / \mathrm{n}$ energy range to account for an energy dependence. 
Lastly, the answer to the discrepancy between the $\mathrm{Co} / \mathrm{Ni}$ isotopic measurements and the higher-energy elemental abundance ratios could be astrophysical in nature. It is possible that the phenomena behind GCR acceleration differ at low and high energies. However, the fact that the interpretation of the ACE/CRIS Co/Ni elemental abundance ratio, made using the Webber \& Gupta curves, differs from the clear ACE/CRIS isotopic measurement strongly suggests that the discrepancy lies with the calculations of expected $\mathrm{Co} / \mathrm{Ni}$ ratio.

All in all, improved models of SN nucleosynthesis and GCR propagation, as well as new total and partial cross sections for $\mathrm{Ni}$ and $\mathrm{Co}$ at higher energies will permit a cleaner interpretation of the $\mathrm{Co} / \mathrm{Ni}$ elemental ratio. Those improved models, combined with measurements with further improved resolution and statistical accuracy will permit a better determination of the time between nucleosynthesis and acceleration of cosmic rays above $1 \mathrm{GeV} /$ nucleon.

TABLE 1. TIGER Co/Ni Ratios

\begin{tabular}{lcc}
\hline \multicolumn{1}{c}{ Measurement } & Abundance & Error \\
\hline $\begin{array}{l}\text { From fit at } \\
\text { TIGER }\end{array}$ & 0.128 & $+/-0.051$ \\
$\begin{array}{l}\text { After efficiency } \\
\text { correction }\end{array}$ & 0.114 & $+/-0.045$ \\
$\begin{array}{l}\text { Top of } \\
\text { Atmosphere }\end{array}$ & 0.093 & $+/-0.037$ \\
\hline
\end{tabular}

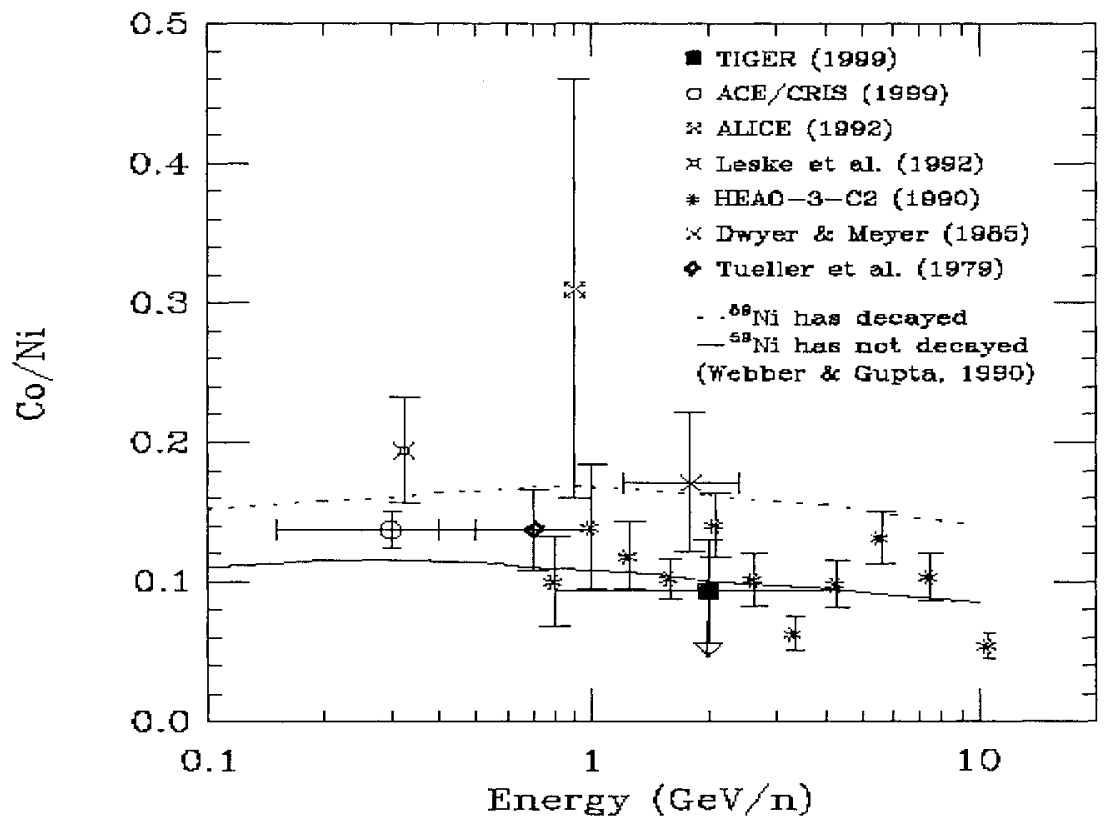

FIGURE 4. Recent measurements of the elemental abundance of $\mathrm{Co} / \mathrm{Ni}$. The solid and dotted lines are from Webber \& Gupta [14]. Square - TIGER measurement from this work; open circle - ACE/CRIS [7]; asterisks - HEAO-3-C2 [2]; fancy cross ALICE [3]; fancy square - Leske et al. [6]; cross - Dwyer \& Meyer [1]; diamond - Tueller et al. [11].

This work was supported in part by NASA under grant NAG5-5110.

\section{REFERENCES}

1. Dwyer, R., and Meyer, P., ApJ, 294, 441 (1985).

2. Engelmann, J.J, et al., $A \& A$, 233, 96 (1990).

3. Esposito, J.A., et al., APH, 1, 33 (1992).

4. Israel, M.H., et al., ICRC, 1, 323 (1979).
5. Lawrence, D.J., et al., NIM, 420, 402 (1999).

6. Leske, R.A., et al., ApJL, 390, 99 (1992).

7. Lijowski, M., et al., ICRC, 3, 5 (1999).

8. Soutoul, A., et al., ApJ, 219, 753 (1978).

9. Sposato, S.H., et al., SCIFI 97, p. 527 (1997).

10. Sposato, S.H., Washington University Ph.D.

Thesis, St. Louis, MO (1999)

11. Tueller, J., et al., ApJ, 228, 582 (1979).

12. Webber, W.R., et al., ICRC, 1, 325 (1987).

13. Webber, W.R. et al., ApJ, 508, 940 (1998).

14. Webber, W.R., \& Gupta, M., ApJ, 384, 608 (1990)

15. Wiedenbeck, M.E., et al., ApJL, 523, 61 (1999). 